\title{
Curso en línea de educación en valores
}

\author{
Values education online course
}

\author{
Marisol Rodríguez \\ macrodriguez4@gmail.com \\ https://orcid.org/0000-0003-0732-8669 \\ Yolanda Rodríguez \\ Yrodriguezuc888@gmail.com \\ https://orcid.org/0000-0002-7422-9073 \\ Universidad de Carabobo, Valencia, Venezuela
}

Recibido: 17/12/2020

Aceptado: 15/01/2021

\section{Resumen}

La investigación tuvo como propósito el diseño de un curso en línea para la Educación en Valores. El estudio se enmarcó en un diseño no experimental transaccional de campo de tipo descriptivo, bajo la modalidad de proyecto factible. La población estuvo integrada por 70 estudiantes siendo la muestra de tipo intencional de $30 \%$ es decir 20 sujetos. La técnica para la recolección de datos, fue un cuestionario tipo encuesta; con escala Likert y respuestas policotómicas. Para calcular la confiabilidad se manejó el Coeficiente de Alpha de Cronbach. La validación del instrumento se realizó a través de juicios de expertos, tanto en contenido, como en diseño y metodología. El cual se utilizó para el análisis de datos a través de técnicas estadísticas de carácter descriptivo, donde cada variable se analizó individualmente. Se concluyó que un curso en línea es una herramienta importante e interactiva de aprendizaje sin límites de espacio y tiempo, que conecta a los estudiantes desde diferentes escenarios permitiendo la consolidación del conocimiento.

Palabras clave: Curso en Línea, estrategias, tecnología, aprendizaje.

\section{Abstract}

The initiative of an online learning course is to understand another way to materialize the concept of teaching in the educational process. It is about optimizing this relationship between students and teachers to ensure that all actors in the educational act can be participants and collaborators. The purpose of the research was to design an online course for Education in Values. Based on Gagné's Information Processing Theory and Negrete's Theory of Universal Values. Framed in a non-experimental, 
transactional descriptive field design, under the modality of a feasible project. The population was made up of 70 students, the intentional sample being $30 \%$, that is, 20 subjects. The technique for data collection was a survey type questionnaire; with Likert scale and polycotomic responses. Cronbach's Alpha Coefficient was used to calculate reliability. The validation of the instrument was carried out through expert judgments, both in content, design and methodology. Which was used for data analysis through descriptive statistical techniques, where each variable was analyzed individually? It was concluded that an online course is an important and interactive learning tool without limits of space and time, which connects students from different settings, allowing the consolidation of knowledge.

Keywords: Online Course, strategies, technology, learning.

\section{Introducción}

Los nuevos entornos de enseñanza y aprendizaje exigen nuevos roles en docentes y estudiantes. La perspectiva tradicional en educación superior, por ejemplo, del docente como única fuente de información y sabiduría y de los estudiantes como receptores pasivos debe dar paso a papeles bastante diferentes. La misión del docente en entornos ricos en información es la de facilitador, la de guía y consejero, la de creador de hábitos y destrezas en la búsqueda, selección y tratamiento de la información. Los estudiantes, por su parte, deben adoptar un papel mucho más importante en su formación, no sólo como meros receptores pasivos de lo generado por el profesor, sino como agentes activos en la búsqueda, selección, procesamiento y asimilación de la información la cual implica comunicación y cumple una función primordial que contribuye a la globalización.

Por otra parte, los nuevos canales abren un frente en los conocimientos y destrezas del docente. Debe utilizarlos y ayudar a utilizarlos a sus estudiantes, como una herramienta al servicio de su propia autoformación. De hecho, cada vez en más universidades, los profesores atienden sus tutorías también por correo electrónico, tienen páginas web con los programas de sus asignaturas y las lecturas recomendadas y utilizan los nuevos canales como medio de comunicación y para reforzar la interacción del grupo de estudiantes entre sí, se trata de ampliar el tipo de experiencias formativas utilizando medios que van a encontrar en los diferentes ámbitos de su vida profesional y que forman parte de la cultura tecnológica que lo impregna todo.

La digitalización y los nuevos soportes electrónicos están dando lugar a nuevas formas de almacenar y presentar la información que superan en determinados contextos las formas tradicionales de la explicación oral, la pizarra, los apuntes y el manual.

Para la UNESCO (2011), las prácticas de las TIC dependen de su integración exitosa en las salas de clases con la implementación de estructuras de ambientes de aprendizaje no-tradicionales, de la unión de nuevas tecnologías con nuevas 
pedagogías en ambientes virtuales de aprendizaje, del desarrollo de clases socialmente activas, del fomento de la interacción cooperadora, el trabajo cooperativo y el trabajo grupal. De igual manera, la UNESCO (2011) considera que uno de los factores de mayor impacto se fundamenta en los estándares de competencias TIC para el profesor, desde el enfoque de alfabetización digital o tecnológica y profundización del conocimiento hasta llegar a la creación del conocimiento.

Del mismo modo, Claro (2010) se refiere a estos estándares como consistentes con los objetivos de desarrollo del milenio definidos por las Naciones Unidas y especifican los cambios que implica para cada componente del sistema educativo: política, currículum y evaluación, pedagogía, uso de la tecnología, organización y administración escolar, y desarrollo profesional docente. (p.10)

Díaz (2011), en su ensayo las TIC en la educación y los retos que enfrentan los docentes señala, la región latinoamericana, con base en los análisis de los expertos en el tema, se encuentra un claro rezago no sólo en las posibilidades de acceso en condiciones de equidad a dichas tecnologías, sino también en relación a sus usos pedagógicos. Uno de los desafíos más importantes se refiere a la tarea docente. Las nuevas exigencias a la profesión docente demandan que sean precisamente los profesores los responsables de la alfabetización tecnológica de sus estudiantes y del dominio de una diversidad de competencias requeridas en el contexto de las demandas de la sociedad del conocimiento. La cuestión es ¿están preparados los docentes para ello?, ¿se está haciendo lo debido para asegurar una formación docente apropiada?

García (2004), plantea los recursos tecnológicos les permiten a los docentes ser capaces de posesionarse en su desempeño como corresponsables del aprovechamiento, de la conceptualización y del papel que juegan los recursos tecnológicos en los procesos de enseñanza y aprendizaje. Señala que el uso de recursos tecnológicos educativos ha incrementado y, por ende, las nuevas tecnologías de la información y comunicación han generado grandes cambios e impactos en la manera de enseñar y aprender, es decir, en la educación de los seres humanos. La implementación de las tecnologías de la información y comunicación, en el ámbito educativo, abre las puertas al desarrollo de habilidades que le permiten al estudiante explorar, crear y compartir nuevos aprendizajes o conocimientos mediante la utilización de estos recursos.

Loureiro (2006), "obliga a los ciudadanos a contar con nuevas competencias y conocimientos, lo que evidencia la necesidad de actualizar los procesos educativos tradicionales" y por lo mismo se ha visto la necesidad de incorporar las TIC en dichos procesos, partiendo de las necesidades del entorno y de los mismos educandos.

De esta manera, se ha observado como en las diferentes instituciones de educación superior los docentes han ido incorporando herramientas tecnológicas como recursos didácticos para la presentación y el manejo de la información al estudiante, así como 
para la adquisición de conceptos, habilidades, actitudes y destrezas. Es de suma importancia la implementación de las nuevas TIC en la educación en los diferentes entornos educativos y especialmente los dirigidos para la educación superior, sobre todo para el desarrollo de sus diferentes programas y mucho más en la actualidad que se está requiriendo su implementación como apoyo didáctico en un gran número de las asignaturas que conforman el currículo de las diferentes etapas de la Educación Superior.

\section{Referentes teóricos}

El basamento teórico relacionado en este trabajo está sustentado en la Teoría del Procesamiento de la Información de Gagné (1974), quien comienza a elaborar su teoría del aprendizaje con la finalidad de que ésta sirva de base para una teoría de la instrucción. Los fundamentos de esta teoría se hallan en los elementos básicos que, para él, constituyen el aprendizaje. Para lograr ciertos resultados es preciso conocer las condiciones internas que van a intervenir en el proceso y las condiciones externas que van a favorecer un aprendizaje óptimo.

En este sentido, el autor plantea un modelo de procesamiento de información el cual fue organizado en cuatro partes específicas: los procesos de aprendizaje (primera fase) consisten en el cambio de una capacidad o disposición humana, que persiste en el tiempo y que no puede ser atribuido al proceso de maduración. El cambio se produce en la conducta del individuo, posibilitando inferir que se logre a través del aprendizaje. Para el logro de éste, surge un mecanismo interno durante el proceso de aprendizaje la cual obedece a la validez de información hacia el cerebro, que posteriormente se almacena en una memoria de corto o largo alcance. Una vez que la información ha sido registrada en cualquiera de las dos memorias, no son diferentes como estructuras, sino en "momentos", ésta puede ser retirada o recuperada, sobre la base de los estímulos externos que hagan necesaria esa información. Asimismo, destaca que existen mecanismos internos en el estudiante en el acto de aprender, las cuales denomina: fases del aprendizaje (segunda fase) motivación, comprensión, adquisición, retención, recuperación, generalización, ejecución y realimentación. Esta fase está intrínsecamente relacionada con los estímulos que recibe el educando, los cuales cumplen un papel importante en los procesos de enseñanza y aprendizaje debido a que cada una cumple una función específica. En dicho proceso donde el educando recibe información ingresa a la memoria transitoria denominada también corto alcance donde fijará el conocimiento, si este conocimiento fue realmente consolidado el estudiante puede recuperarlo a través de la memoria de largo alcance.

De no ocurrir la fijación del conocimiento quedará en la memoria de corto alcance y al no utilizarla se olvidará. En cuanto a las capacidades aprendidas corresponde a su tercera fase, desde la óptica de anteriormente citado, existen cinco clases de capacidades que pueden ser aprendidas. Las mismas son el punto de partida de un proceso muy importante que es el de la evaluación, deberán ser las mismas capacidades aprendidas las que se evaluarán para determinar el éxito del aprendizaje. 
Estas son: destrezas motoras, información verbal, destrezas intelectuales, actitudes y estrategias cognoscitivas. La cuarta y última fase, son los tipos de aprendizaje que a su vez son parte del proceso (primera fase). Los tipos describen las formas en que este se puede dar en el individuo. Entre ellos son: aprendizajes de signos y señales, Respuestas operantes, en cadena, Asociaciones verbales, de discriminaciones múltiples, de conceptos y aprendizaje de principios.

En resumen, Gagné (1974), plantea que en la situación de aprendizaje se identifica inicialmente cuatro elementos:

- El estudiante.

- La situación de estimulación

- La conducta de entrada (lo que ya está en memoria)

- La conducta final que se espera del estudiante.

La primera incluye los procesos de aprendizaje, es decir cómo el individuo aprende y cual son los postulados hipotéticos sobre los cuales se construye la teoría; la segunda parte analiza los resultados del aprendizaje que aprende el estudiante; la tercera parte trata de las condiciones del aprendizaje, es decir los eventos facilitadores del aprendizaje y finalmente la cuarta corresponde a los tipos de aprendizaje que puede adquirir el estudiante. Esta distinción ha sido expresada por Gagne y Dick (1983) de la siguiente forma:

Las teorías y modelos de la instrucción intentan relacionar sucesos específicos referidos a la instrucción con procesos de aprendizaje y con resultados de aprendizaje, apoyándose en el conocimiento generado por la investigación y la teoría del aprendizaje. Las teorías de la instrucción son a menudo prescriptivas en el sentido de que aspiran a identificar las condiciones de instrucción que optimizarán el aprendizaje, la retención y la transferencia de lo aprendido. Para ser clasificadas como teorías, estas formulaciones deben proporcionar, como mínimo, una descripción racional de las relaciones causales entre procedimientos de enseñanza empleados y sus consecuencias comportamentales en la mejora de las realizaciones humanas...aunque haya desacuerdo sobre los requerimientos específicos de tal teoría. (p. 264)

\section{Los valores universales}

Negrete (2014), señala que el desarrollo humano es un proceso de descubrimiento, crecimiento, humanización, conquista de la libertad; representa el esfuerzo de hombres y mujeres por conquistarse a sí mismos mediante la iluminación de la inteligencia y el fortalecimiento de la voluntad, y con apertura, resultado del amor a los demás. Estos valores forman un perfil de hombre que encarna convicciones y creencias funcionales para un determinado ideal de sociedad integrada por la conducta colectiva, el comportamiento humano social y los valores deseables. 
En una sociedad como la nuestra, los valores expresan el perfil de hombre que resulta de un contexto cultural y un concepto de nación. Los valores se manifiestan en actitudes y grandes habilidades de aplicación múltiple que en conjunto son las que permiten lograr las capacidades y competencias requeridas. ¿Por qué de aplicación múltiple? Porque facilitan el trabajo científico con base en el desarrollo de un pensamiento inquisitivo, lógico y crítico, el manejo de lenguajes y el dominio de disciplinas. Un desarrollo humano integral, finalmente, cubre todas las posibilidades de crecimiento.

Por lo general, la escuela o la empresa se preocupan por los aspectos cognoscitivos o racionales. Valores universales: son el conjunto de normas de convivencia válidas en un tiempo y época determinada. Los valores, ante la solución del dilema anterior, no son sencillos. Esto se debe a que en ocasiones se confrontan valores importantes y entran en conflicto. ¿Cuáles? El derecho a la vida y a la salud, el respeto a la propiedad privada, la observancia de las leyes entre otros. Un concepto decisivo y, estrechamente ligado a los valores, es el de la actitud. ¿Qué son las actitudes? Son tendencias adquiridas que predisponen a una persona a reaccionar de un modo determinado ante un objeto, persona, suceso o situación, y actúa en consecuencia. Una de éstas es la "opinión", que es la manifestación pública, por lo general verbalizada, de un sentimiento o creencia.

Expresa un valor o una actitud. Otro concepto relevante es el "hábito" que es un comportamiento o modo de proceder regular y adquirido por la repetición de actos similares.

Valores Universales

Amistad: es el afecto o estimación entre personas que les permite establecer vínculos más estrechos de convivencia.

Amor: es un principio de unión entre los elementos que forman el universo; una manifestación de los hombres hacia el bien y la belleza absoluta.

Bondad: es una cualidad de una cosa o persona que la voluntad considera como un fin deseable tendiente a lo bueno.

Confianza: actitud de esperanza hacia una persona o cosa; sentimiento de seguridad en uno mismo; acto de fe.

Fraternidad: es la unión y buena correspondencia entre los hombres.

Honor: es el sentimiento profundo de la propia dignidad moral del hombre.

Honradez: es la cualidad que nos hace proceder con rectitud e integridad.

Justicia: es dar a cada quien lo que se merece, según sus obras.

Libertad: es obrar con libre albedrío; es hacer lo que uno desea sin dañar a nadie. La libertad física es limitada y sólo el pensamiento es infinitamente libre.

Paz: es el conjunto de actos de unión o concordia que hacen posible la convivencia armoniosa entre los miembros de una sociedad o familia.

Respeto: es la consideración especial hacia las personas en razón de reconocer sus cualidades, méritos, situación o valores particulares. 
Responsabilidad: es el deber de asumir las consecuencias de los actos que uno ejecuta sin que nadie obligue

Solidaridad: es una responsabilidad mutua contraída por varias personas, que nos hace colaborar de manera circunstancial en la causa de otros.

Tolerancia: actitud abierta hacia posturas u opiniones diferentes a la propia.

Valentía: es la cualidad que nos permite enfrentar con decisión y sin dudar todos los actos de nuestra vida.

Verdad: es la conformidad o acuerdo de lo que se dice con lo que se siente, se piensa o hace.

El desarrollo de valores y actitudes

El aprendizaje de los valores y de las actitudes es un proceso lento y gradual en donde influyen distintos factores y agentes. Aunque los rasgos de la personalidad y el carácter de cada quien son decisivos en su adquisición, también desempeñan un papel indiscutible las experiencias personales previas, el medio donde se crece, las actitudes que transmiten las personas significativas, a información y las vivencias escolares, los medios masivos de comunicación entre otros. La primera que aparece en el desarrollo del individuo durante la infancia, es la moral heterónoma, es decir, lo que un poder o una ley externos determinan como adecuado o no. (Negrete, 2014)

En ese tipo de moral los niños se sienten obligados a cumplir las normas morales porque así lo exige una autoridad superior. Las personas no hacen una elección libre, consciente o responsable, ni juzgan las normas morales por el valor que tienen en sí mismas, sino por la fuerza de la jerarquía o autoridad de quien las impone de por intención de los actos que generan independientemente, de quien los impone.

\section{Educación en Línea}

Se entiende por educación en línea a aquella en la que los docentes y estudiantes participan en un entorno digital a través de las nuevas tecnologías y de las redes de computadoras, haciendo uso intensivo de las facilidades que proporciona Internet y las tecnologías digitales. La literatura de la educación a distancia para cursos donde se usa correspondencia, video, la transmisión por televisión y por satélite, no necesariamente describen el proceso incluido en los cursos en línea Schwier, (2003). Un desarrollo histórico de la educación en línea y un análisis del desarrollo histórico del Internet y sus conexiones deben existir para entender cómo y porqué ha crecido tan rápido."

Si bien algunos autores como García (1999), consideran que se trata de una evolución de la educación a distancia que se apoya en recursos digitales para desplegar los procesos de enseñanza y aprendizaje utilizando herramientas propias de las tecnologías de la información y la comunicación (TIC) para otros pedagogos, como Caldeiro (2013), la educación en línea es una modalidad con características propias 
que se diferencia tanto de la educación a distancia como de la educación presencial Desde esta perspectiva, la educación en línea es caracterizada como un modelo pedagógico que promueve, mediante el uso de tecnologías digitales, ambientes propicios para el diálogo y actividades grupales buscando favorecer, incluso, la creación de vínculos interpersonales entre los participantes.

De este modo, se entiende que las propuestas de educación en línea usualmente implementadas a través plataformas o entornos digitales para la gestión de cursos (conocidos como Learning Management System) incluyen actividades pedagógicas que para su realización requieren que los estudiantes colaboren entre sí trabajando de forma conjunta. Esta colaboración entre educandos se produce sin que medien encuentros presenciales y es posible a partir de los diálogos sincrónicos y asincrónicos que se producen a través de las herramientas de comunicación telemática, generalmente, incluidas en el mismo software sobre el que se desarrolla el curso y que tienden a promover la documentación automática de los intercambios entre los participantes.

De esta forma, Caldeiro (2013) señala también que mientras que en la educación a distancia la enseñanza, se apoya principalmente en la distribución de materiales didácticos (digitalizados o en otros formatos no digitales) en la educación en línea son fundamentales las actividades que los estudiantes deben resolver para aprender.

En la actualidad, la educación en línea está convirtiéndose en una opción accesible para realizar estudios de bachillerato, universitarios y de especialización o posgrado para aquellas personas que por su ubicación geográfica o por cuestiones laborales les resulta muy difícil acudir a una institución presencial, además de ser una estrategia educativa basada en la aplicación de tecnología al aprendizaje sin limitación del lugar, tiempo, ocupación o edad de los estudiantes. Con el tiempo el campo de esta disciplina se ha profesionalizado cada vez más hasta abarcar no solo estudios formales, sino también cursos de actualización y capacitación laboral en múltiples profesiones y oficios. Algunos rasgos fundamentales de esta modalidad:

La inmersión de estudiantes en un entorno digital a través del cual interactúan mientras transcurre los procesos de aprendizaje.

* El uso pedagógico de las Tecnologías de la Información y Comunicación

* La inclusión de actividades que proponen trabajo colaborativo entre los estudiantes

La utilización de materiales didácticos en diversos formatos digitales

Por vincular sus orígenes con la educación a distancia, los docentes que trabajan en cursos de educación en línea, suelen recibir el nombre de tutores, ya que su función primordial es la de acompañar y asistir al estudiante a lo largo de su proceso de aprendizaje en el contexto de un programa que carece de un espacio de físico como en la educación presencial. Por las características generales de la educación en línea, el papel que docente/tutor suele asumir e incluye una serie de funciones que, 
dependiendo del curso, pueden variar en su nivel de complejidad. Este proceso de acompañamiento y asistencia, nos permite mencionar algunos rasgos del tutor virtual señalados por Medina, E. (2014), tales como enfocarse en el ambiente de aprendizaje, promover el liderazgo y la innovación, promover la interacción comunicativa y aplicar criterio para juzgar el éxito; valorando así los actos concretos experimentados.

\section{Recorrido metodológico}

La presente investigación se realizó bajo el paradigma cuantitativo, de campo, no experimental y descriptiva, con modalidad de Proyecto factible el cual: "consiste en la elaboración y desarrollo de una propuesta de un modelo operativo viable para solucionar problemas, requerimientos 0 necesidades de organizaciones o grupos sociales; puede referirse a la formulación de políticas, programas, tecnologías, métodos o procesos". UPEL (2015:7).

En este caso, se presenta el diseño de un Curso en Línea como estrategia didáctica tecnológica para la Educación en Valores de los estudiantes del Curso Introductorio de la Facultad de Ciencias de la Educación de la Universidad de Carabobo. Se empleó la técnica de encuesta, y el instrumento fue un cuestionario impreso con escala tipo Likert: Siempre (S), A veces (AV), Nunca (N). Según Sabino (2002), es una técnica de adquisición de información de interés sociológico, mediante un cuestionario previamente elaborado, a través del cual se puede conocer la opinión o valoración del sujeto seleccionado en una muestra sobre un asunto dado en este caso el diseño de un curso en línea de educación en valores.

La validación se realizó a través de juicio de expertos y probada su confiabilidad mediante el estadístico Alfa de Cronbach obteniendo 0,92 (alta confiabilidad). Posteriormente, el instrumento referido, fue aplicado a 20 sujetos de estudio, quienes son estudiantes de la Facultad de Educación de la Universidad de Carabobo, campus Bárbula estado Carabobo los cuales dieron su consentimiento para la obtención de los datos.

Para el cumplimiento de los objetivos propuestos en este estudio, se siguieron las siguientes etapas:

Detección del problema.

Revisión bibliográfica

Redacción del planteamiento de problema.

Elaboración de: antecedentes, bases teóricas, y fundamentación legal

Estructura del marco metodológico

Diseño del instrumento de recolectar los datos y de la guía

Validación del instrumento.

Consentimiento Informado para la obtención de los datos

Aplicación de pruebas pilotos. 
Verificación de la Confiabilidad del instrumento.

Organización de los resultados.

Análisis y discusión de los resultados.

Formulación de las conclusiones y recomendaciones

Diseño del curso en línea para la educación en valores

\section{Análisis e interpretación de los resultados}

Los datos se presentan a través de tablas estadísticas en donde se describen cuáles fueron las cuantificaciones de las frecuencias absolutas y relativas de las respuestas dadas por los entrevistados que participaron de la muestra a través de un instrumento denominado estudio por encuesta, para la variable independiente, esto se realiza para cada uno de los supuestos a partir de los ítems que lo definen. Posteriormente cuando se tabularon los datos se construye un gráfico representativo con un diagrama de barras, esto con la finalidad de visualizar y comparar las opiniones emitidas por los encuestados a los planteamientos realizados en los ítems.

Variable

Curso en línea

Dimensión: Asincronía

Indicador: Chat. Ítems $\mathbf{N}^{\circ}$ 13.- Con la implementación de un chat en línea se puede evaluar a un estudiante. TABLA N 1 . Distribución porcentual. Resultados obtenidos del instrumento aplicado a los Estudiantes.

Gráfico № 1. Asincronía

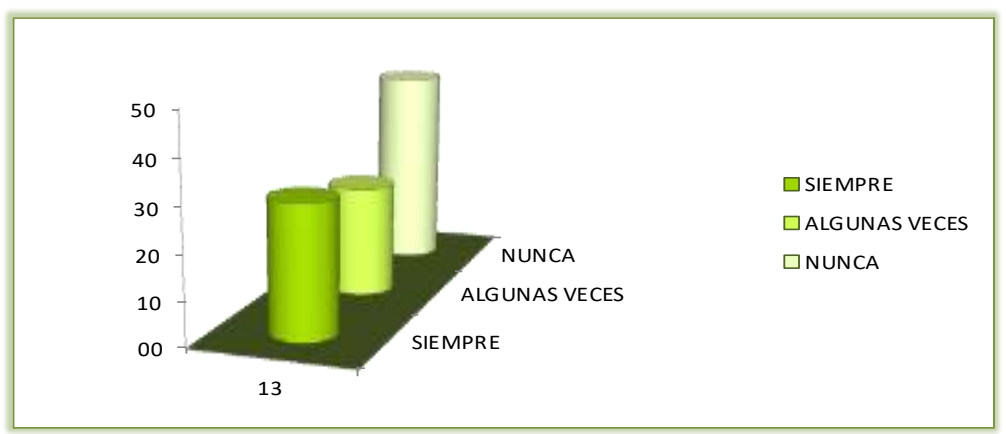

Rodríguez, Y. (2019)

Interpretación:

En el gráfico $\mathrm{N}^{\circ} 2$, se presentan los resultados obtenidos cuando se solicitó a los estudiantes encuestados su consideración sobre si los chats sobre la comunicación asertiva son estrategias para el aprendizaje. Se observa en el gráfico mencionado, que 
un $30 \%$, de los estudiantes encuestados, dicen que "siempre", el $25 \%$ expresó que "algunas veces" y $45 \%$ "nunca", lo que quiere decir que los estudiantes consideran que con la implementación de un chat en línea se puede evaluar a un estudiante.

\section{Conclusiones y recomendaciones}

A manera de conclusión se puede decir que el diseño y ejecución de un curso en línea de aprendizaje le permite tanto a estudiantes y docentes involucrados en el acto educativo interactuar de manera interactiva, a distancia, es una estrategia novedosa y actualizada. Hoy por hoy, el sistema educativo se organiza respondiendo a una visión de la educación muy limitada, al otorgar la responsabilidad de la formación de las personas únicamente a la educación formal y a las instituciones del sistema educativo; es decir, a las escuelas, centros de educación secundaria, universidades, centros de formación superior, entre otros. La educación no se entiende como una responsabilidad compartida por el conjunto de la sociedad sino como una responsabilidad a gestionar por los sistemas educativos y los profesionales que trabajan en ellos. Satisfacer las necesidades educativas de la población y hacer frente a los desafíos que plantea el nuevo escenario social, económico, político y cultural marcado por la globalización, las nuevas tecnologías de la información y comunicación y el cambio a una economía basada en el conocimiento, precisa ampliar el concepto de educación y aceptar que esta es responsabilidad de toda la sociedad y que requiere de un compromiso compartido de esta y de la comunidad de la que forma parte el sistema educativo Asimismo, exige un cambio profundo que replantee no sólo lo qué se enseña sino también cómo se enseña, dónde se enseña y para qué se enseña. Los cursos en línea (CL) son una respuesta a esta visión más amplia de la educación, en la medida en que buscan transformar las prácticas pedagógicas y la organización habitual de las instituciones educativas formales, al mismo tiempo que movilizar los recursos ajenos a éstas poniéndolos al servicio de la educación y de la formación de las personas.

\section{Referencias}

Caldeiro, G. (2013). El aprendizaje en red y el trabajo colaborativo en entorno mediados por tecnología http://www.pent.org.ar/institucional/publicaciones/aprendizaje-red-trabajo-

(2013). colaborativo-entornos-mediados-por-tecnologia.

Claro, Magdalena. (2010). La incorporación de tecnologías digitales en educación. Modelos de identificación de buenas prácticas. Naciones Unidas. https://www.cepal.org/es/publicaciones/

Díaz, B. (2011). Las TIC en la educación y los retos que enfrentan los docentes. (UNAM, México. http://www.oei.es/historico/metas2021/libro.html.

Gagne, R.M. y Briggs, S.J. (1974). Principios de diseño instruccional. Nueva York, Holt, Rinehart y Winston (Traducción castellana: Planificación de la Enseñanza, México: Trillas 
Gagne, R.M. y Dick, W. (1983) Psicología de la Instrucción. Annual Review of Psychology.

García, A. (1999). Historia de la Educación a Distancia. RIED. Revista Iberoamericana de Educación a Distancia", volumen 2, no 1. http://www.utpl.eduec/ried/images/pdfs/vol2

García, J. (2004). Ambientes con recursos tecnológicos escenarios para la construcción de procesos pedagógicos. San José. Costa Rica. EUNED.

Hernández Sampieri, Fernández y Baptista (2014) Metodología de la Investigación. México: McGraw Hill.

Loureiro, A. (2006). La escuela en la sociedad de redes. Una introducción a las tecnologías de la información y de la comunicación en la educación. Lectura y Vida: Revista latinoamericana de lectura, 27(2), 73-74. Recuperado el 28 de Julio de 2014de la base de datos virtual Proquest.

Medina, E. (2014). Virtualización didáctica de la planificación instruccional. Dirección de Medios y publicaciones. Universidad de Carabobo. Venezuela.

Negrete, L (2014) Los Valores Universales. Consulta 05/10/2019. https://www.uv.mx/psicologia/files/2014/11/valores-universales.pdf

Sabino, C. (2002). El Proyecto de Investigación Científica. Caracas: Panapo.

Schwier, R. y Misanchuk, E. (2003). Instrucción multimedia interactiva. Englewood Cliffs, N.J. Publicaciones de tecnología educativa.

UNESCO. (2011). Normas UNESCO sobre competencias en TIC para docentes.

Universidad Pedagógica Experimental Libertador. (2015) Manual de Tesis de Grado y Maestría y Tesis Doctorales. Venezuela. 